\title{
Criteria for Predicting the Formation of Single-Phase High-Entropy Alloys
}

\author{
M. Claudia Troparevsky, ${ }^{1}$ James R. Morris, ${ }^{1,2}$ Paul R. C. Kent, ${ }^{3,4}$ Andrew R. Lupini, ${ }^{1}$ and G. Malcolm Stocks ${ }^{1}$ \\ ${ }^{1}$ Materials Science and Technology Division, Oak Ridge National Laboratory, \\ Oak Ridge, Tennessee 37831, USA \\ ${ }^{2}$ Department of Materials Science and Engineering, \\ University of Tennessee, Knoxville, Tennessee 37996, USA \\ ${ }^{3}$ Center for Nanophase Materials Sciences, Oak Ridge National Laboratory, \\ Oak Ridge, Tennessee 37831, USA \\ ${ }^{4}$ Computer Science and Mathematics Division, Oak Ridge National Laboratory, \\ Oak Ridge, Tennessee 37831, USA
}

(Received 5 November 2014; published 31 March 2015)

\begin{abstract}
High-entropy alloys constitute a new class of materials whose very existence poses fundamental questions regarding the physical principles underlying their unusual phase stability. Originally thought to be stabilized by the large entropy of mixing associated with their large number of components (five or more), these alloys have attracted attention for their potential applications. Yet, no model capable of robustly predicting which combinations of elements will form a single phase currently exists. Here, we propose a model that, through the use of high-throughput computation of the enthalpies of formation of binary compounds, predicts specific combinations of elements most likely to form single-phase, highentropy alloys. The model correctly identifies all known single-phase alloys while rejecting similar elemental combinations that are known to form an alloy comprising multiple phases. In addition, we predict numerous potential single-phase alloy compositions and provide three tables with the ten most likely five-, six-, and seven-component single-phase alloys to guide experimental searches.
\end{abstract}

DOI: 10.1103/PhysRevX.5.011041

Subject Areas: Materials Science

The term high-entropy alloy (HEA) has come to signify nontraditional alloy systems composed of five or more elements at, or near, equiatomic ratio that form random, single-phase solid solutions on simple underlying facecentered-cubic (fcc) and body-centered-cubic (bcc) lattices [1-11]. HEAs stand in sharp contrast to traditional metal alloys that are typically based on one or two primary elements and where addition of further alloying elements often leads to the formation of new phases. Clearly, the existence of HEAs poses important questions regarding the driving mechanism for their unexpected stability and how to identify the specific combinations of elements that are most likely to form a single-phase HEA.

Although there are several proposals regarding the stability of HEAs, much of the existing work uses semiempirical approaches based, for example, on HumeRothery rules, thus focusing on the differences of the atomic sizes $(\delta)$, electronegativities $(\Delta \chi)$, and electron-toatom ratio $(e / a)$ [12-17]. Some approaches utilize calculation of phase diagrams methods [18], while others consider $\delta$, the enthalpy of mixing $\left(\Delta H_{\text {mix }}\right)$, and the ideal entropy of mixing of the alloys to develop criteria for the

Published by the American Physical Society under the terms of the Creative Commons Attribution 3.0 License. Further distribution of this work must maintain attribution to the author(s) and the published article's title, journal citation, and DOI. phase stability $[1,12]$. For example, in the work of Guo et al. [14], the use of $\delta$ and $\Delta H_{\text {mix }}$ as independent variables clearly separates solid-solution phases from amorphous phases but does not necessarily isolate intermetallic compounds from either one of these phases. In addition, in the work of Otto et al. [11], there are atomic substitutions to the solid-solution $\mathrm{CrMnFeCoNi}$ alloy that are specifically chosen to follow the Hume-Rothery rules in respect of $\delta$, $\Delta \chi$, and crystal structure yet do not form a single-phase solid solution. Useful as many of these attempts to encapsulate features of the underlying bonding mechanisms have been, it is clear that a model that can robustly predict, out of all of the elements in the periodic table, which combinations of elements can form an HEA, and which cannot, has yet to emerge. As the number of possible combinations of elements increases factorially with the number of components, easily exceeding $10^{5}$ even for fivecomponent systems and restricting the search to the simple and transition metals, it is clear that an unguided search for new HEAs is unfeasible. Given that a number of HEAs also possess unusual combinations of strength, ductility, thermal stability, corrosion, and wear resistance [19-25] that make them candidates for technological applications, developing a predictive model assumes even greater significance.

Here, we propose a simple criterion, based on enthalpy considerations, to predict which elemental combinations are most likely to form a single-phase HEA. Enthalpies are 
evaluated via first-principles "high-throughput" densityfunctional-theory (DFT) calculations [26-30] of the energies of formation of binary compounds and therefore require no experimental or empirically derived input. The model correctly accounts for the specific combinations of metallic elements that are known to form single-phase HEAs, while rejecting similar combinations that have been tried and shown not to be single phase.

Within our method, a set of elements will form a singlephase alloy if the enthalpy of formation of all the possible binary compounds formed by combinations of these elements falls within a specified range. This range is such that the compounds are neither too stable, leading to precipitation of that phase, nor too unstable, indicating immiscibility of the constituent elements. Remarkably, considering only the formation of binary compounds is sufficient to accurately predict the formation of singlephase alloys and correctly identifies closely related compositions that form multiple phases. In order to assess the formation of single-phase alloys, we construct a $30 \times 30$ enthalpy matrix (see the Supplemental Material [31]) containing the lowest enthalpies of formation of all binary combinations of the elements: $\mathrm{Mg}$; $\mathrm{Al}$; all the $3 d, 4 d$, and $5 d$ transition metals, except $\mathrm{Tc}$ and $\mathrm{Lu}$, with only $\mathrm{La}$ included from the lanthanides. An $18 \times 18$ subset is shown in Fig. 1.

In order to compare the stability of competing compounds, we should ideally compare their Gibbs free energies. However, multicomponent solid-solution alloys typically present rather small (and similar) enthalpies of formation, while ordered compounds typically have very small entropic terms. To compare the stability of the competing phases, we utilize, as a first approximation, only the contributions $\left(-T \Delta S_{\text {mix }}\right)$ from mixing entropy $\left(\Delta S_{\text {mix }}\right)$ for solid-solution alloys and only the enthalpy of formation $\left(\Delta H_{f}\right)$ for the ordered compounds. Even with these simplifying approximations, finding the enthalpies of formation of all possible (binary, ternary, quaternary, etc.) ordered intermetallics that can result from a given alloy's component species, and hence their extremal envelope as a function of composition (the convex hull), remains a very formidable task when the number of alloying species is large. However, if we consider only pairwise combinations, we can take advantage of the fact that the convex hulls of a large number of binary alloy systems have already been obtained via high-throughput DFT calculations [28-30]. That it is sufficient to consider only the binary phases is, no doubt, due in part to the slow diffusion rate of the alloying species at typical annealing temperatures, which makes the formation of more complex compounds, such as ternaries and quaternaries, much less likely.

The values in the enthalpy matrices are obtained mainly from "data mining" of the binary alloy library of Curtarolo et al. and the alloy database of Widom and co-workers [28,32]. We independently recalculate many $\Delta H_{f}$ for these binaries and obtain good agreement with the databases. We also calculate $\Delta H_{f}$ for two $\sigma$ phases ( $\mathrm{MnCr}$ and $\mathrm{MnMo}$ ). We obtain values of some laves $\left(\mathrm{TiCr}_{2}, \mathrm{ZrCr}_{2}\right.$, and $\left.\mathrm{HfCr}_{2}\right)$

\begin{tabular}{|c|c|c|c|c|c|c|c|c|c|c|c|c|c|c|c|c|c|c|}
\hline $\mathrm{Ti}$ & V & $\mathrm{Cr}$ & $\mathrm{Mn}$ & $\mathrm{Fe}$ & Co & $\mathbf{N i}$ & $\mathrm{Cu}$ & $\mathrm{Zn}$ & $\mathrm{Nb}$ & Mo & $\mathbf{R u}$ & Rh & Pd & $\mathrm{Ta}$ & $\mathbf{w}$ & $\operatorname{Re}$ & Os & \\
\hline Ti & 37 & -372 & -277 & -418 & -386 & -435 & -147 & -198 & 11 & -167 & -763 & -790 & -646 & 31 & -82 & -189 & -713 & $\mathrm{Ti}$ \\
\hline 37 & 0 & -88 & -286 & -176 & -199 & -250 & 13 & -51 & -56 & -127 & -321 & -393 & -275 & -122 & -97 & -148 & -361 & V \\
\hline-372 & -88 & 0 & -110 & -8 & 5 & -30 & 108 & 44 & -47 & 42 & 4 & -129 & -82 & -130 & 26 & 4 & -22 & $\mathrm{Cr}$ \\
\hline-277 & -286 & -110 & 0 & 9 & -19 & -115 & 29 & -25 & -153 & -138 & -15 & -188 & -251 & -254 & -92 & -139 & -40 & Mn \\
\hline-418 & -176 & -8 & 9 & 0 & -60 & -97 & 65 & -23 & -2505 & -484 & 41 & -57 & -116 & 3468 & -554 & -25 & 11 & Fe \\
\hline-386 & -199 & 5 & -19 & -60 & 0 & -21 & 54 & -58 & -178 & -52 & 52 & 12 & -10 & -253 & -84 & -72 & 34 & Co \\
\hline 435 & -250 & -30 & -115 & -97 & -21 & 0 & -6 & -256 & -316 & -100 & 40 & 2 & -6 & -746 & -116 & -116 & 32 & $\mathrm{Ni}$ \\
\hline-147 & 54 & 108 & 29 & 65 & 54 & -6 & 0 & -92 & -29 & 83 & 108 & -4 & -126 & 28 & 129 & 83 & 141 & $\mathrm{Cu}$ \\
\hline-198 & -51 & 44 & -25 & -23 & -58 & -256 & -92 & 0 & -160 & -42 & -150 & -391 & -571 & -88 & 58 & 8 & 21 & $\mathrm{Zn}$ \\
\hline Nb & -56 & -47 & -153 & -2505 & -150 & -316 & -29 & -160 & 0 & -133 & -249 & -548 & -435 & -10 & -76 & -202 & -276 & $\mathrm{Nb}$ \\
\hline-167 & -127 & 42 & -136 & -484 & -52 & -100 & 83 & -42 & -133 & 0 & -57 & -248 & -100 & -193 & -8 & -2 & -52 & Mo \\
\hline Ru & -321 & 4 & -15 & 41 & 52 & 40 & 108 & -150 & -249 & -57 & 0 & -8 & 47 & -332 & -66 & -87 & -16 & $\mathbf{u}$ \\
\hline 90 & -393 & -129 & -188 & -57 & 12 & 2 & -4 & -391 & -548 & -248 & -8 & 0 & 37 & -611 & -273 & -181 & -8 & Rh \\
\hline 646 & -275 & -82 & -251 & -116 & -10 & -6 & -126 & -571 & -435 & -100 & 47 & 37 & 0 & -480 & -123 & -57 & 67 & Pd \\
\hline 31 & -122 & -130 & -254 & -3468 & -253 & -746 & 28 & -88 & -10 & -193 & -332 & -611 & -480 & 0 & -114 & -226 & -330 & Ta \\
\hline-82 & -97 & 26 & -92 & -554 & -84 & -116 & 129 & 58 & -76 & -8 & -66 & -273 & -123 & -114 & 0 & 7 & -56 & $\mathbf{W}$ \\
\hline-189 & -148 & 4 & -139 & -25 & -72 & -116 & 83 & 8 & -202 & -2 & -87 & -181 & -57 & -226 & 7 & 0 & -89 & 6 \\
\hline 713 & -361 & -22 & -40 & 11 & 34 & 32 & 141 & 21 & -276 & -52 & -16 & -8 & 67 & -330 & -56 & -89 & 0 & Ds \\
\hline $\mathrm{Ti}$ & v & $\mathrm{Cr}$ & $M n$ & $\mathrm{Fe}$ & Co & $\mathrm{Ni}$ & $\mathrm{Cu}$ & $\mathrm{Zn}$ & $\mathrm{Nb}$ & Mo & $\mathbf{R u}$ & $\mathbf{R h}$ & $\mathbf{P d}$ & $\mathrm{Ta}$ & $\mathbf{w}$ & $\mathrm{Re}$ & Os & \\
\hline
\end{tabular}

Energy scale (meV/atom)

\begin{tabular}{|l|l|l|l|l|ll|l|l|l|l|}
\hline & & & & & & & & & \\
\hline
\end{tabular}

FIG. 1. Enthalpy matrix. Calculated enthalpies of formation of the lowest energy structures of binary compounds relative to phase separation into pure elements. The numbers in bold blue font have been calculated with respect to the solid solution. 
and $\mu$ phases (MnCr, MnMo, FeNb, FeMo, FeTa, and FeW) from Chen et al. and Ansara et al., respectively [33]. All the calculations are properly spin polarized, a necessary condition for the accurate treatment of the energetics of elemental metals and intermetallic compounds involving the midperiod $3 d$ transition elements $\mathrm{Cr}, \mathrm{Mn}, \mathrm{Fe}, \mathrm{Co}$, and Ni. Entries in Fig. 1 represent the $\Delta H_{f}$ of the lowest energy structure of each binary compound relative to phase separation into pure elements. Here, it is important to note that each entry involves the results of DFT calculations for hundreds of compounds, considering different compositions, lattices, and elemental decorations within each lattice type; thus, the full enthalpy matrix represents the distillation of many tens of thousands of such calculations. In addition, all calculations are based on essentially identical computational methodologies; in particular, the same exchange-correlation functional (generalized gradient approximation-Perdew, Burke and Ernzerhof) is used in all of the DFT calculations, thereby minimizing the relative errors among the $\Delta H_{f}$ values of Fig. 1 [34]. In addition, this exchange-correlation functional is known to give a superior description of the equilibrium volumes and energetics of metals and alloys to that of the local density approximation (LDA), especially for the magnetic elements where LDA even gives the wrong ground-state structure of $\mathrm{Fe}$ [35].

The values of $\Delta H_{f}$ that determine the optimal range for the formation of single-phase HEAs are rationalized as follows. The minimum value of the range is set by the ideal entropy of mixing as $-T_{\mathrm{ann}} \Delta S_{\text {mix }}$, where $T_{\mathrm{ann}}$ is the annealing temperature used in the experimental setting. For instance, for the alloys of Otto et al. [11], the annealing temperature used $\left(T_{\mathrm{ann}}=1000 \mathrm{~K}\right)$ corresponds to an enthalpy of $-138.7 \mathrm{meV} /$ atom. For the case of the bcc alloys made by Senkov et al. [36], $T_{\mathrm{ann}}=1673 \mathrm{~K}$, which corresponds to a $\Delta H_{f}=-232 \mathrm{meV} /$ atom. Clearly, the former is consistent with $\mathrm{CrMnFeCoNi}$ forming a single phase in that the lowest energy of formation of any binary chosen from $\mathrm{Cr}, \mathrm{Mn}, \mathrm{Fe}, \mathrm{Co}$, and $\mathrm{Ni}$ taken from the enthalpy matrix of Fig. 1 is $-115 \mathrm{meV} /$ atom. The upper limit corresponds to the largest value of $\Delta H_{f}$ for which the alloy does not phase separate due to the immiscibility of any pair of elements. The upper limit of the enthalpy range $(37 \mathrm{meV}$ ) is chosen to include all known singe-phase alloys and it is consistent with the thermodynamic model presented by Poletti and Battezzati [16]. It is worth noting that $T_{\text {ann }}$ could be replaced by some critical temperature ( $\left.T_{\text {crit }}\right)$, below which diffusion is sufficiently slow that the enthalpic driving force is insufficient to result in phase decomposition on a realizable time scale. While different choices of the limits of allowable $\Delta H_{f}$ lead to different allowed combinations of elements that will form a HEA, it should be compatible with whatever annealing temperature would be required to homogenize the particular alloy. For example, the temperature may be some fraction of the average melting temperature of the constituent metals (for example,
$T_{\text {crit }} \sim 0.6 T_{M}$ ). It turns out such a criterion is already quite consistent with the annealing temperature used experimentally. For example, the $T_{\text {ann }}$ used by Otto et al. $(1000 \mathrm{~K})$ for $\mathrm{CrMnFeCoNi}$ [11] corresponds to a $T_{\text {crit }}=0.55 T_{M}$, while the same $T_{\text {ann }}$ for CrPdFeCoNi [10] corresponds to $T_{\text {crit }}=0.54 T_{M}$. For the bec alloys $\mathrm{VNbMoTaW}$ and NbMoTaW, $T_{\text {ann }}=1673 \mathrm{~K}$, which corresponds to a $T_{\text {crit }}$ of $0.56 T_{M}$ and $0.53 T_{M}$, respectively.

Using our matrix, we can now rapidly examine all possible single-phase alloys that result from a specified number of components by comparing the extremal values of the enthalpies of the binaries to $-T_{\mathrm{ann}} \Delta S_{\text {mix }}$. Using the enthalpy limits specified above, our approach predicts all presently known single-phase HEAs. In addition, this approach provides predictions of the most probable five-, six-, and seven-component alloys, thereby providing guidance to the search for new alloys. A list of these systems is given in the Supplemental Material. In addition, the model can be used to determine the best elemental choice to add to an existing alloy; e.g., for $\mathrm{CrMnFeCoNi}$, the best choice is Os.

Beyond these predictions, our model offers the flexibility of adding (or customizing) different criteria to search for new alloys. For example, one can add a desired range for the density of the alloy, sort them by price per $\mathrm{kg}$, or even modify the range of acceptable enthalpies. For example, choosing the lower limit to be some specific fraction of $T_{M}$ (say, $T_{\text {crit }}=0.55 T_{M}$ ) as suggested above would not significantly change our predictions.

As an example of how the above approach works, Table I shows predictions for two classes of binaries:

TABLE I. Prediction of multiple and single-phase alloys. Both tables indicate which one-component (diagonal entries) and twocomponent (upper off-diagonal entries) additions to the base alloy (FeCoNi or NbMoTa) are likely to form single-phase solid solutions.

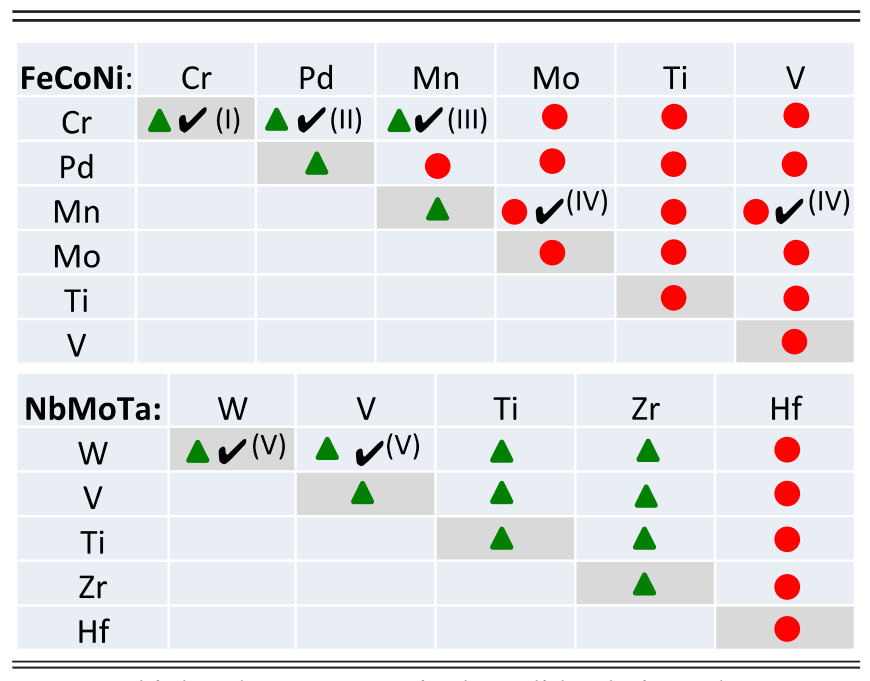

$=$ multiple phases $\boldsymbol{\Delta}=$ single solid-solution phase $\boldsymbol{V}=$ experimentally confirmed. (I): Ref. [15] (II): Ref. [10] (III): Ref. [9] (IV): Ref. [11], and (V): Ref. [36]. 
TABLE II. Five-, six-, and seven-component alloys for which the enthalpy of formation of the binary compounds are $-138<\Delta H_{f}<37 \mathrm{meV} /$ atom (range 1) and $-232<\Delta H_{f}<$ $37 \mathrm{meV}$ (range 2).

\begin{tabular}{lccc}
\hline \hline & Five components & Six components & Seven components \\
\hline All & $1.69911 \times 10^{5}$ & $7.36281 \times 10^{5}$ & $2.62958 \times 10^{6}$ \\
Range 1 & 179 & 69 & 0 \\
Range 2 & 269 & 93 & 17 \\
\hline \hline
\end{tabular}

one based on additions to the face-centered-cubic FeCoNi system, one based on the body-centered-cubic NbMoTa system. The matrices indicate which one-component (diagonal entries) and two-component (upper off-diagonal entries) additions to the base alloy are likely to form singlephase solid solutions, and which are not. Where possible, experimental checks have been given. As an example, adding either $\mathrm{Mn}$ or $\mathrm{Pd}$ to $\mathrm{CrFeCoNi}$ is predicted to yield single phases, consistent with experiment. However, adding both $\mathrm{Mn}$ and Pd is predicted to form multiple phases, due to the strong Mn-Pd interaction (Fig. 1). Similarly, while Mn and $\mathrm{Cr}$ may be added, $\mathrm{Mn}$ and $\mathrm{V}$ may not, agreeing with experiment.

Table II contains the number of possible single-phase alloys for five-, six-, and seven-component alloys for two enthalpy ranges. The minimum values of these ranges reflect annealing temperatures of 1000 and $1673 \mathrm{~K}$. The number of possible combinations of elements is very large, rising to $2.629 \times 10^{6}$ for seven-component alloys. Despite this great number, our model predicts that no sevencomponent single-phase alloys would form if we consider a range of $-138 \mathrm{meV} /$ atom $<\Delta H_{f}<37 \mathrm{meV} /$ atom, and only 17 alloys would form if we extend the range to $-232 \mathrm{meV} /$ atom $<\Delta H_{f}<37 \mathrm{meV} /$ atom. Table II provides a list of possible additions to existing alloys and indicates the ones that have been verified experimentally.

In comparing our model with experiment, we also examine the $\Delta H_{f}$ of the lowest ordered binary intermetallic compounds containing the elements $\mathrm{Ti}, \mathrm{V}, \mathrm{Cr}, \mathrm{Mn}, \mathrm{Fe}$, $\mathrm{Co}, \mathrm{Cu}$, and $\mathrm{Mo}$ (Fig. 2). These data span all of the systems (CrMnFeCoNi, CrMnFeTiNi, MoMnFeCoNi, VMnFeCoNi, CrMnVCoNi, and $\mathrm{CrMnFeCoCu}$ ) included in the work of Otto et al. [11], an experimental study designed to demonstrate that configurational entropy alone is insufficient to stabilize HEAs. While CrMnFeCoNi is found to form a single phase, substitution of one of the component elements by $\mathrm{Ti}, \mathrm{Mo}, \mathrm{V}$, or $\mathrm{Cu}$ (consistent with the Hume-Rothery rules) results in the precipitation of intermetallic phases despite the fact that the entropy of mixing should be unchanged by any of the substitutions. As can be seen from Fig. 2, the binaries comprised of $\mathrm{Cr}, \mathrm{Mn}$, $\mathrm{Fe}, \mathrm{Co}$, and $\mathrm{Ni}$ all have relatively small values of $\Delta H_{f}$ consistent with it being a single-phase system. On the other hand, binaries containing V, Ti, or Mo display strongly

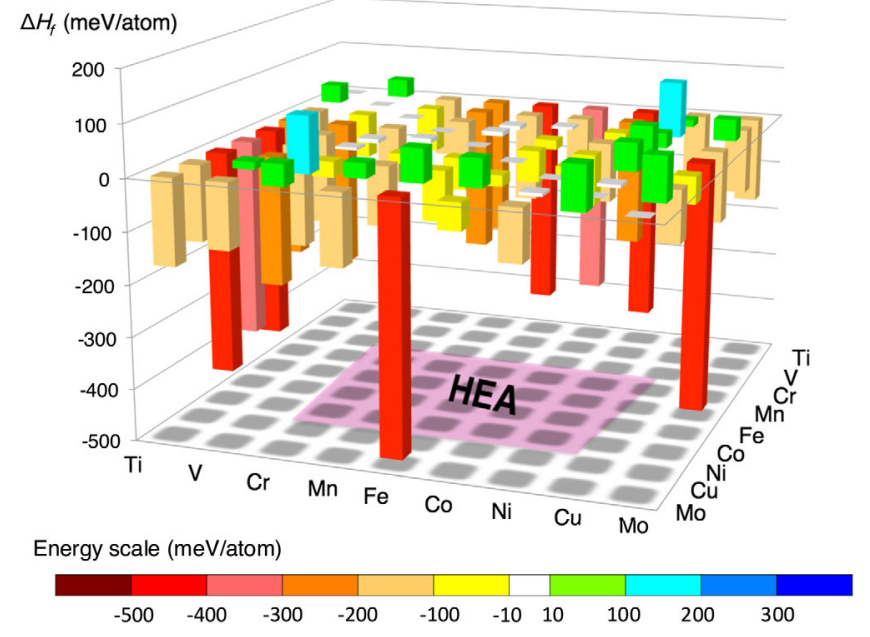

FIG. 2. Graphical representation of the enthalpies of formation of the binary compounds that could form from the alloys studied in Ref. [11].

negative $\Delta H_{f}$, favoring ordered intermetallic formation, again consistent with the multiphase phase behavior found by Otto et al. The binaries containing $\mathrm{Cu}$ present the opposite trend; their $\Delta H_{f}$ are positive and much larger than typical (Fig. 2). In the case of $\mathrm{CrMnFeCoCu}$, the instability of this HEA is driven by the immiscibility of $\mathrm{Cu}$ with the other metallic species.

We now consider the HEAs NbMoTaW and VNbMoTaW first made by Senkov et al. [36]]. These refractory alloys have high melting points, thus widening the range of allowable enthalpies of formation. Examining $\Delta H_{f}$ of the possible binaries for these alloys (Fig. 1), the lowest value corresponds to MoTa at $-193 \mathrm{meV} /$ atom. This value corresponds to a minimum annealing temperature of $1391 \mathrm{~K}$, which is indeed lower than the annealing temperature of $1673 \mathrm{~K}$ used in Ref. [36].

Finally, our results are also consistent with Wang et al. [2] where it was found that $\mathrm{AlCrFeCoNiCu}$ alloys contain an intermetallic NiAl phase, despite earlier characterization as an ideal HEA. Consideration of our enthalpy matrix reveals that the $\Delta H_{f}$ of the NiAl binary is $-677 \mathrm{meV} /$ atom, which would require an annealing at $4881 \mathrm{~K}$ to form the HEA. Thus, in accordance with our method, $\mathrm{AlCrFeCoNiCu}$ should form multiple phases with $\mathrm{Ni}$ and $\mathrm{Al}$, providing the basis of the intermetallic phase, as found experimentally.

An alternative to our model is that the HEA formers are themselves unusually stable energetically. To test this hypothesis, we perform DFT calculations [37,38] of the $\Delta H_{f}$ for supercell models of the disordered phase. We consider two well-studied alloys that form a single phase $\mathrm{CrFeCoNi}$ and $\mathrm{CrMnFeCoNi}$, and one that forms a second phase CrMnFeTiNi [11]. The computed $\Delta H_{f}$ values are $72.9,91.1$, and $87.8 \mathrm{meV} /$ atom, respectively. From these results, it is clear that there are no substantive differences in 
the $\Delta H_{f}$ values between the three systems that could account for their differing stabilities. It is even the case that $\Delta H_{f}$ for $\mathrm{CrMnFeTiNi}$, which precipitates a second phase, is less than that of $\mathrm{CrMnFeCoNi}$, which does not. These results demonstrate that consideration of the enthalpy of formation of the HEA itself is not a predictor of single-phase compositions.

It is worth noting that within our method, we utilize the enthalpies of formation relative to the phase separation into the pure elements. An arguably better approach would be to reference the $\Delta H_{f}$ of the binary compounds relative to that of the corresponding binary solid solution. For most binaries, changing the reference will make little difference, as the enthalpies of formation of solid solutions are typically small. But, there are a few exceptions in which the solid solution is itself very stable. Clear examples are binaries between $\operatorname{Re}$ and some early $3 d, 4 d$, and $5 d$ elements. For instance, our calculated $\Delta H_{f}$ of ReTa and $\mathrm{ReV}$ bcc solid solutions are -177 and $-211 \mathrm{meV} /$ atom, respectively. These unusually stable binary solid solutions make the ordered phases predicted by DFT calculations much less stable. If we did not include the Re solid solutions in our calculations, we would not be able to predict the formation of some Re-containing alloys. Notably, these extremely stable intermetallics are also absent from the experimental binary phase diagram $[39,40]$.

Our results clearly contradict the entropy alone hypothesis that increasing the number of components will necessarily yield a more stable alloy. While the entropy of mixing does increase and could therefore widen the range mentioned in the previous paragraphs, increasing the number of components also increases the probability of finding a very stable ordered (often binary) intermetallic, thus decreasing the number of possible single-phase solid solutions.

In summary, we investigated the stability of multicomponent alloys and presented a method to determine the formation of single-phase HEA alloys. The method is simple and easy to use, and has so far shown excellent agreement with reported experimental results. While we have concentrated on delineating the combinations of elements that can yield ideal solid-solution alloys, our enthalpy matrix can also be used to search for specific elemental additions to single-phase alloys in order to precipitate a particular second phase that can then be manipulated to produce a desired microstructure with, for example, improved yield strength. Indeed, there are already examples of attempts to do exactly this based on the addition of $\mathrm{Al}$ to $3 d$ transition-metal-based alloys such as $\mathrm{CrFeCoNi}$. A clean example of second phase precipitation is shown in the work of Manzoni et al. [41] who do indeed find that the addition of $\mathrm{Al}$ to $\mathrm{CrFeCoNi}$ results in phase separation into a $\mathrm{Ni}$-Al-rich matrix phase and $\mathrm{Cr}$-Fe-rich precipitates. As noted earlier, this finding is completely in line with our enthalpy matrix and the observation that the
NiAl-based intermetallic phase is the most stable binary compound between $\mathrm{Al}$ and any of the alloy's base elements.

Research was supported by the U.S. Department of Energy, Basic Energy Sciences, Materials Sciences and Engineering Division (M.C.T., G. M. S., J.R.M., and A.R. L.) and through a user project supported by ORNL's Center for Nanophase Materials Sciences (CNMS), which is sponsored by the Scientific User Facilities Division (PRCK), Basic Energy Sciences, U.S. Department of Energy. Calculations were performed on ORNL, BES funded, clusters and at the National Energy Research Scientific Computing Center (NERSC).

[1] J. W. Yeh, S. K. Chen, S. Lin, J. Gan, T. Chin, T. T. Shun, C. Tsau, and S. Chang, Nanostructured High-Entropy Alloys with Multiple Principle Elements: Novel Alloy Design Concepts and Outcomes, Adv. Eng. Mater. 6, 299 (2004).

[2] Y. Wang, B.S. Li, and H.Z. Fu, Solid Solution or Intermetallics in a High-Entropy Alloy, Adv. Eng. Mater. 11, 641 (2009).

[3] C. Hsu, W. Wang, W. Tang, S. Chen, and J. W. Yeh, Microstructure and Mechanical Properties of New $\mathrm{AlCo}_{x} \mathrm{CrFeMo}_{0.5} \mathrm{Ni}$ High-Entropy Alloys, Adv. Eng. Mater. 12, 44 (2010).

[4] X. Ye, M. Ma, W. Liu, L. Li, M. Zhong, Y. Liu, and Q. Wu, Synthesis, and Characterization of High-Entropy Alloy $\mathrm{Al}_{x} \mathrm{FeCoNiCuCr}$ by Laser Cladding, Adv. Mater. Sci. Eng. 2011, 1 (2011).

[5] M. A. Hemphill, T. Yuan, G. Y. Wang, J. W. Yeh, C. W. Tsai, A. Chuang, and P.K. Liaw, Fatigue Behavior of $\mathrm{Al}_{0.5} \mathrm{CoCrCuFeNi}$ High Entropy Alloys, Acta Mater. 60, 5723 (2012).

[6] R. Sriharitha, B.S. Murty, and R. S. Kottada, Phase Formation in Mechanically Alloyed $\mathrm{Al}_{x} \mathrm{CoCrCuFeNi}$ $(x=0.45,1,2.5,5$ mol) High Entropy Alloys, Intermetallics 32, 119 (2013).

[7] A. Manzoni, H. Daoud, S. Mondal, S. van Smaalen, R. Völkl, U. Glatzel, and N. Wanderka, Investigation of Phases in $\mathrm{Al}_{23} \mathrm{Co}_{15} \mathrm{Cr}_{23} \mathrm{Cu}_{8} \mathrm{Fe}_{15} \mathrm{Ni}_{16}$ and $\mathrm{Al}_{8} \mathrm{Co}_{17} \mathrm{Cr}_{17} \mathrm{Cu}_{8} \mathrm{Fe}_{17} \mathrm{Ni}_{33}$ High Entropy Alloys and Comparison with Equilibrium Phases Predicted by Thermo-Calc, J. Alloys Compd. 552, 430 (2013).

[8] Y. Zhang, T. T. Zuo, Y. Q. Cheng, and P. K. Liaw, HighEntropy Alloys with High Saturation Magnetization, Electrical Resistivity, and Malleability, Sci. Rep. 3, 1455 (2013).

[9] B. Cantor, I. T. H. Chang, P. Knight, and A. J. B. Vincent, Microstructural Development in Equiatomic Multicomponent Alloys, Mater. Sci. Eng. A 375-377, 213 (2004).

[10] M. S. Lucas, L. Mauger, J. A. Muñoz, Y. Xiao, A. O. Sheets, S. L. Semiatin, J. Horwath, and Z. Turgut, Magnetic and Vibrational Properties of High-Entropy Alloys, J. Appl. Phys. 109, 07E307 (2011).

[11] F. Otto, Y. Yanga, H. Beia, and E. P. George, Relative Effects of Enthalpy and Entropy on the Phase Stability of Equiatomic High-Entropy Alloys, Acta Mater. 61, 2628 (2013). 
[12] S. Guo, C. Ng, J. Lu, and C. T. Liu, Effect of Valence Electron Concentration on Stability of fcc or bcc Phase in High Entropy Alloys, J. Appl. Phys. 109, 103505 (2011).

[13] S. Guo and C. T. Liu, Phase Stability in High Entropy Alloys: Formation of Solid-Solution Phase or Amorphous Phase, Prog. Nat. Sci. Mater. Int. 21, 433 (2011).

[14] S. Guo, Q. Hu, C. Ng, and C. T. Liu, More Than Entropy in High-Entropy Alloys: Forming Solid Solutions or Amorphous Phase, Intermetallics 41, 96 (2013).

[15] M. Ren, B. Li, and H. Fu, Formation Condition of Solid Solution Type High-Entropy Alloy, Trans. Nonferrous Met. Soc. China 23, 991 (2013).

[16] M.G. Poletti and L. Battezzati, Electronic and Thermodynamic Criteria for the Occurrence of High Entropy Alloys in Metallic Systems, Acta Mater. 75, 297 (2014).

[17] Y. Zhang, T. T. Zuo, Z. Tang, M. C. Gao, K. A. Dahmen, P. K. Liaw, and Z. P. Lu, Microstructures and Properties of High-Entropy Alloys, Prog. Mater. Sci. 61, 1 (2014).

[18] D. B. Miracle, J. D. Miller, O. N. Senkov, C. Woodward, M. D. Uchic, and J. Tiley, Exploration and Development of High Entropy Alloys for Structural Applications, Entropy 16, 494 (2014).

[19] K. B. Zhang and Z. Fu, Effects of Annealing Treatment on Phase Composition and Microstructure of $\mathrm{CoCrFeNiTiAl}_{x}$ High-Entropy Alloys, Intermetallics 22, 24 (2012).

[20] B. Gludovatz, A. Hohenwarter, D. Catoor, E. H. Chang, E. P. George, and R. O. Ritchie, A Fracture-Resistant High-Entropy Alloy for Cryogenic Applications, Science 345, 1153 (2014).

[21] H. Tong, M. Chen, S. Chen, J. W. Yeh, T. Shun, S. Lin, and S. Chang, Mechanical Performance of the $\mathrm{Al}_{x} \mathrm{CoCrCuFeNi}$ High-Entropy Alloy System with Multiprincipal Elements, Metall. Mater. Trans. A 36A, 1263 (2005).

[22] M. Tsai, C. Wang, C. Tsai, W. Shen, J. W. Yeh, J. Gan, and W. Wu, Thermal Stability and Performance of NbSiTaTiZr High-Entropy Alloy Barrier for Copper Metallization, J. Electrochem. Soc. 158, H1161 (2011).

[23] Y. Y. Chen, T. Duval, U. D. Hung, J. W. Yeh, and H. C. Shih, Microstructure and Electrochemical Properties of High Entropy Alloys-A Comparison with Type-304 Stainless Steel, Corros. Sci. 47, 2257 (2005).

[24] J. Wu, S. Lin, J. W. Yeh, S. Chen, Y. Huang, and H. Chen, Adhesive Wear Behavior of $\mathrm{Al}_{x} \mathrm{CoCrCuFeNi}$ High-Entropy Alloys as a Function of Aluminum Content, Wear 261, 513 (2006).

[25] K. C. Hsieh, C. F. Yu, W. T. Hsieh, W. R. Chiang, J. S. Ku, J. H. Lai, C. P. Tu, and C. C. Yang, The Microstructure and Phase Equilibrium of New High Performance High-Entropy Alloys, J. Alloys Compd. 483, 209 (2009).

[26] S. Curtarolo, D. Morgan, K. Persson, J. Rodgers, and G. Ceder, Predicting Crystal Structures with Data Mining of Quantum Calculations, Phys. Rev. Lett. 91, 135503 (2003).

[27] D. Morgan, G. Ceder, and S. Curtarolo, Computational Crystal Structure Prediction with High-Through-put Ab Initio and Data Mining Methods, J. Miner. Metal. Mater. Soc. 56, 70 (2004).
[28] S. Curtarolo et al., AFLOWLIB.ORG: A Distributed Materials Properties Repository from High-Throughput Ab Initio Calculations, Comput. Mater. Sci. 58, 218 (2012).

[29] G. L. W. Hart, S. Curtarolo, T. B. Massalski, and O. Levy, Comprehensive Search for New Phases and Compounds in Binary Alloy Systems Based on Platinum-Group Metals, Using a Computational First-Principles Approach, Phys. Rev. X 3, 041035 (2013).

[30] S. Curtarolo, G. L. W. Hart, M. B. Nardelli, N. Mingo, S. Sanvito, and O. Levy, The High-Throughput Highway to Computational Materials Design, Nat. Mater. 12, 191 (2013).

[31] See Supplemental Material at http://link.aps.org/ supplemental/10.1103/PhysRevX.5.011041 for the full 30-element enthalpy matrix displaying the enthalpies of formation of the lowest energy structure of each binary compound.

[32] The data from the alloy database of Widom and co-workers are based on electronic density-functional calculations using vasp performed by Mihalkovic and Widom, et al., http://alloy.phys.cmu.edu.

[33] X. Q. Chen, W. Wolf, R. Podloucky, and P. Rogl, Ab Initio Study of Ground-State Properties of the Laves Phase Compounds $\mathrm{TiCr}_{2}, \mathrm{ZrCr}_{2}$, and $\mathrm{HfCr}_{2}$, Phys. Rev. B 71, 174101 (2005); I. Ansara, T. G. Chart, A. F. Guillermet, F. H. Hayes, U. R. Kattner, D. G. Pettifor, N. Saundres, and K. Zeng, Thermodynamic Modelling of Solutions and Alloys, CALPHAD: Comput. Coupling Phase Diagrams Thermochem. 21, 171 (1997).

[34] The only exceptions are the $\mu$ phases, which are very stable compounds.

[35] C. S. Wang, B. M. Klein, and H. Krakauer, Theory of Magnetic and Structural Ordering in Iron, Phys. Rev. Lett. 54, 1852 (1985); D. J. Singh, W. E. Pickett, and H. Krakauer, Gradient-Corrected Density Functionals: FullPotential Calculations for Iron, Phys. Rev. B 43, 11628 (1991); J. Zhu, X. W. Wang, and S. G. Louie, FirstPrinciples Pseudopotential Calculations of Magnetic Iron, Phys. Rev. B 45, 8887 (1992).

[36] O. N. Senkov, G. B. Wilks, J. M. Scott, and D. B. Miracle, Mechanical Properties of $\mathrm{Nb}_{25} \mathrm{Mo}_{25} \mathrm{Ta}_{25} \mathrm{~W}_{25}$ and $\mathrm{V}_{20} \mathrm{Nb}_{20} \mathrm{Mo}_{20} \mathrm{Ta}_{20} \mathrm{~W}_{20}$ Refractory High-Entropy Alloys, Intermetallics 19, 698 (2011).

[37] G. Kresse and J. Furthmüller, Efficient Iterative Schemes for Ab Initio Total-Energy Calculations Using a Plane-Wave Basis Set, Phys. Rev. B 54, 11169 (1996).

[38] G. Kresse and D. Joubert, From Ultrasoft Pseudopotentials to the Projector Augmented-Wave Method, Phys. Rev. B 59, 1758 (1999).

[39] Binary Alloy Phase Diagrams, edited by T. B. Massalski (ASM, Metals Park, Ohio, 1986).

[40] O. Levy, M. Jahnátek, R. V. Chepulskii, G. L. W. Hart, and S. Curtarolo, Ordered Structures in Rhenium Binary Alloys from First-Principles Calculations, J. Am. Chem. Soc. 133, 158 (2011).

[41] A. Manzoni, H. Daoud, R. Völkl, U. Glatzel, and N. Wanderka, Phase Separation in Equiatomic AlCoCrFeNi High-Entropy Alloy, Ultramicroscopy 132, 212 (2013). 